a result, and its slow-turning wheels mean that errors can take years to correct. The human cost can be great.

Even so, there has never been a miscarriage of justice in the United Kingdom that was attributed to evidence from DNA profiling. By contrast, miscarriages of justice have been uncovered by the later application of DNA technology. In the two appeals described, it is important to note that both Deen and Adams were sent for retrial and were eventually convicted of the crimes of which they had been charged. Only the appeals were controversial; the initial guilty verdicts were upheld.

Truth Machine concludes with a section on fingerprinting - of the dermal kind. Fingerprint examiners identify 'friction ridge details', or marks, at particular positions within the image. If the relative positions of sufficient marks are consistent with the reference sample from a known person, then the fingerprint is deemed to match. Statistics are not used because fingerprints are considered to be unique: geneticist Francis Galton estimated the chance of a random match to be 1 in 64 billion.

However, there is no reason to preclude probabilistic estimation, for example in assessing partial fingerprints. Models exist for doing so, but they require knowledge of the rarity of various marks. Surprisingly, as Lynch and his co-authors point out, such population studies have not yet been done. The challenges are different from those of DNA profiles because a set of dermal fingerprint marks may not be definitive: prints from the same finger may differ simply because of distortion or substrate variations.

Fingerprinting has entered the public psyche as being synonymous with unique identification, and such ideas are difficult to shift once lodged. Applying the same 'fingerprint' phrase to DNA profiles, as in this book's title, implies that they too are unique - but they are not. The term 'DNA fingerprint' should be avoided.

Truth Machine is an interesting read - it illustrates that the controversy of DNA profiling is rooted not in the science, but mainly in the restrictions of the adversarial system. A discussion of how science is applied in jurisdictions that use the inquisitorial system, in countries other than the United States and the United Kingdom, would also be welcome.

Peter Gill is a senior lecturer in chemistry at the University of Strathclyde, Glasgow, UK, and professor of legal medicine at the University of Oslo, Norway. He was principal scientist for the UK Forensic Science Service from 1982 until 2008. e-mail: peter.gill@strath.ac.uk

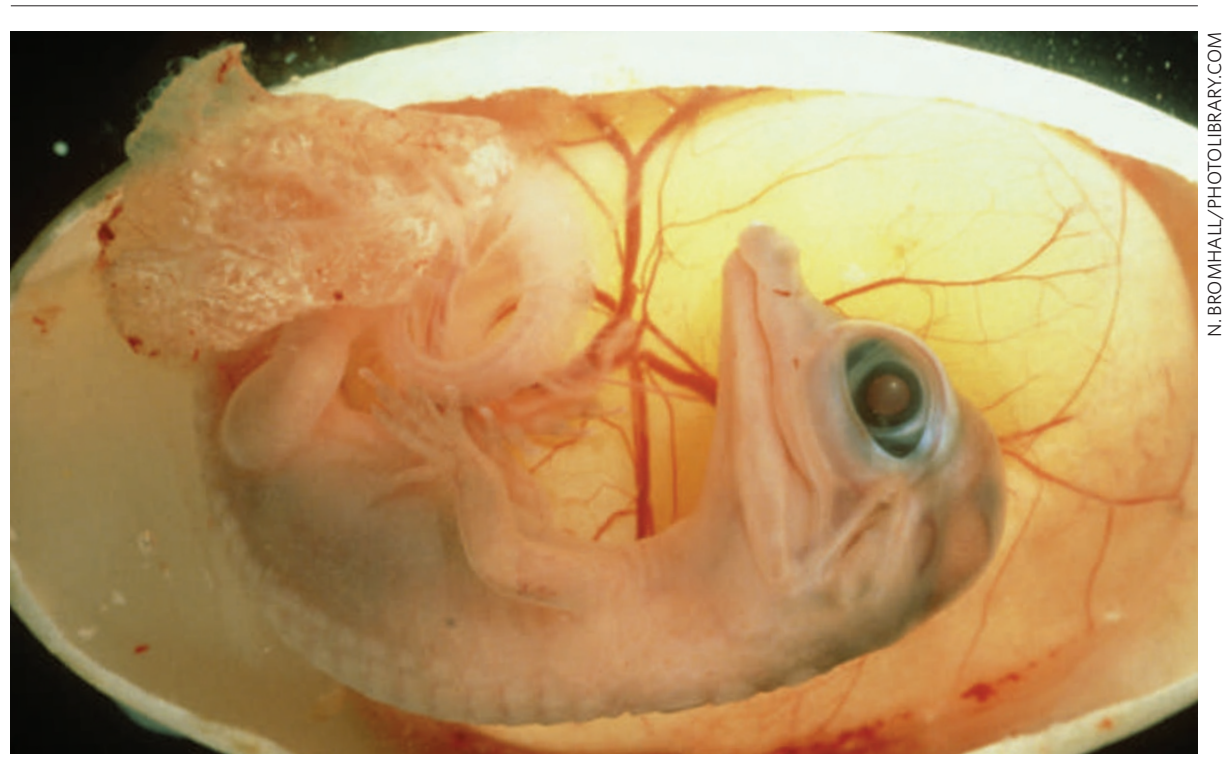

\title{
Evolutionary embryos
}

\section{The Origin of Individuals by Jean-Jacques Kupiec World Scientific Publishing: 2009. 276 pp. $\$ 72, £ 54$}

A central question in biology is how multicellular organisms develop from a single cell and how development is controlled. The standard view is that the process is deterministic, following directives governed by information located in the genome. Molecular biologist Jean-Jacques Kupiec contradicts this picture. In the fascinating The Origin of Individuals he argues that there is no plan, pre-pattern or program encoded in the genome. Instead, cell differentiation and development include a random element.

In the standard view, development is controlled by the binding of protein transcription factors to promoters that activate genes in the DNA. These genes in turn generate proteins, including other transcription factors and signalling molecules that activate yet more genes. A cascade of gene activation results, leading to the proliferation and differentiation of cells that ultimately generates the organism. Assuming that molecular interactions and gene activation are predictable, the development process should be deterministic.

Kupiec argues that this picture is wrong. Gene activation is inherently stochastic, he says, and, therefore, cell differentiation must also be stochastic. Transcription factors attach with certain probabilities to many binding sites in gene promoters, implying that chance plays a dominant role in gene activation and expression. Similarly, cell signalling pathways, and thereby cell interactions, are stochastic, as proteins may bind promiscuously to many partners with various odds. Many interactions and pathways are possible.

As a result of this underlying unpredictability, Kupiec claims, stochastic cellular actions such as cell growth, cell differentiation and cell death must be constrained somehow to ensure that the correct sequence of development occurs. Otherwise, a fertilized egg could grow into any organism.

The problem that ordered biological structures are rarer than the many possible random states led the physicist Erwin Schrödinger in his 1944 book What is Life? to contrast the science of life with physics: in statistical thermodynamics, macroscopic order is generated from disorder, whereas for life to develop, order must be generated from order. Schrödinger introduced the notion of a code script - analogous to a program — contained in the chromosomes, which acts as both plan and operative factor to prevent disorder by guiding the development process.

Kupiec disagrees with the idea of programs. Because of the stochastic nature of protein interaction and gene expression, he says, there can be no Aristotelian form or program to give order to life and ward off entropic chaos and death.

But without a code to follow, how can a particular organism develop from a single cell? Kupiec's radical solution is to apply Darwin's theory of evolution. Put simply, evolution requires two processes - variation and selection. An organism's offspring each varies slightly; natural selection picks out those that 
survive to generate more such organisms, again with their own subtle variations. In the development of an organism the stochastic nature of gene activation and protein interactions permits a vast array of possible developmental outcomes. Darwinian selection, Kupiec argues, constrains development so as to consistently form a particular organism. The local environment of the cell is the selecting agent, choosing which cells survive, differentiate, divide or die. He demonstrates his concept through computer simulations that generate simple patterns of two cell types. Each cell reacts with some probability to its local conditions to determine its next state.

In setting up local environment and metabolic interactions, cells must, however, use signalling protocols or programs that specify how the cells react - even if they involve probabilities. Yet Kupiec claims that because of stochastic protein interactions such programs cannot exist. But even if we allow simple reactive protocols to control any cell's reaction to its local environment, such strategies can produce only simple patterns. They cannot achieve the complex structures and functions generated in many multicellular organisms. Such reactive strategies can, at best, pass on information to determine the cell's next state.

Thus, in Kupiec's proposal, the local environment must host the constraining information necessary to form an organism. But it is not clear how this information could be stored and conveyed. In his view, the environment functions like a complex, external 'homunculus', magically controlling embryonic development at every step. Kupiec also fails to explain why differentiated cells remain stable if gene activation is stochastic, or why cellular control strategies and protocols exist at all.

Kupiec's version of a Darwinian-like cellselection process needs to be robust and invariant. It must be more restrictive than typical Darwinian selection, which permits the formation of a diverse array of organisms and species to form. It must explain why a particular embryo forms, not just any embryo. It must account for the similarity of identical twins; the precision with which the left side mirrors the right in bilaterally symmetrical organisms; and why a mouse differs from a horse or a potato. A further issue is that even if there is local molecular randomness, it need not be passed on to the cell or to the developmental control architecture of the organism. Organisms consistently pass through the same stages during development, irrespective of minor variations in their local and maternal environments.

By treating the genome only as a generator of proteins, Kupiec adopts an implicitly reductionist view of development. But organisms of many species have virtually identical protein structures, yet their control architecture is vastly different, just as a house and a skyscraper can be made of the same parts. Every complex structure needs specific control information to develop, and the only reasonable source of that information is the genome, not some blind local evolutionary selection process. The genome and cell cooperate by means of an epigenetic interpretation system by which control information in the genome is interpreted and executed by the cell. Thus the genome encodes more than protein building-blocks - it contains a hidden control code. Such a feature could explain the vast non-coding regions in the genome; Kupiec prefers to think of these regions as mere space fillers determining geneactivation probabilities.

Kupiec's model also fails to account for global and temporal relationships. Local information is not powerful enough to generate global relationships in an organism - all the more so if it is probabilistic. Because the growth process of an embryo is ordered in time, directives from the genome must be linked to form control networks. The architecture of an organism is complex both spatially and temporally.

Kupiec is a very successful writer, deservedly so. I enthusiastically recommend this courageous book with its iconoclastic viewpoint. The Origin of Individuals is a pleasure to read, presenting complex ideas clearly and effectively. Whether one agrees with him or not, Kupiec's is an inspiring work, a thought-provoking rollercoaster ride through the history of ideas about the origins of ontogeny.

Eric Werner is in the Department of Physiology,

Anatomy and Genetics, and the Computing Laboratory, University of Oxford, South Parks Road, Oxford OX13QX, UK.

e-mail: eric.werner@dpag.ox.ac.uk

\section{Stuffed spectacular}

\author{
Extreme Mammals: The Biggest, Smallest, \\ and Most Amazing Mammals of All Time \\ American Museum of Natural History, \\ New York City \\ Until 3 January 2010
}

Roll up, roll up! See the giant Indricotherium, a plant-eating mammal from Mongolia that weighed as much as four adult African elephants! Gaze at the tiny bumblebee bat, which can hover in place like a hummingbird! Marvel at the wide-eyed sugar gliders, sailing nimbly through the branches! All can be seen in Extreme Mammals, the latest exhibition at the American Museum of Natural History that showcases the largest, smallest, toothiest, brainiest, prickliest, slowest, fastest and lustiest mammals on the planet.

Although its title suggests a circus, the show lacks the pizzazz of a real one. Except for the sugar gliders and the human visitors, none of the mammals in the exhibition is moving or breathing: all are models, skeletons or stuffed.

The museum has more than a million stuffed animals in its collection, and never misses an opportunity to haul them out for display, preferably in one of its beloved dioramas.

Extreme Mammals is a superb example. Among the many immortalized creatures shown are a duck-billed platypus, a fluffy koala, a longtailed howler monkey, a spectacled bear and a striped Tasmanian wolf or thylacine, which went extinct in

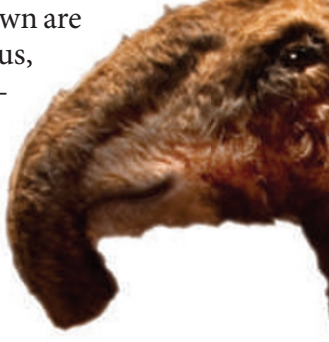

Extinct: South American ungulate Macrauchenia.
1936 when the last of its kind died in an Australian zoo.

One diorama depicts Ellesmere Island now an expanse of frozen tundra some 1,000 kilometres from the North Pole - when it was a warm paradise 50 million years ago, inhabited by the likes of Coryphodon, an animal similar to a hippo but with massive tusks and one of the smallest brains for its size. Surrounded by plywood trees, the foamcarved Coryphodon chews placidly on swamp plants beside a polyurethane pond.

Extreme Mammals offers some amazing sights. Visitors 